\title{
Activation of complement in relation to disease
}

\author{
J. M. B. VERSEY, LINDA SLATER, AND J. R. HOBBS \\ From the Department of Chemical Pathology, Westminster Hospital Medical School, London
}

Activation of the complement system results in the sequential interaction of its protein constituents, producing a series of enzymes from their precursors, each of which acts on the next protein in the chain, forming interprotein complexes of two or more of the cleavage products. The consequences of such activation include increased vascular permeability, attraction of polymorphonuclear leucocytes, the enhancement of phagocytosis, and alterations in cell membranes resulting in lysis and death.

The classical complement system involves nine components labelled $\mathrm{C} 1$ to $\mathrm{C} 9$ which react together in a manner described as a cascade (Macfarlane, 1964). Activation of the system is usually brought about by the combination of an antibody with an antigen, which activates the complement-binding site of the antibody molecule, thought to be situated on the $\mathrm{C}_{\mathrm{H}} 2$ domain. The antigen may be in solution or on a cell surface. The $\mathrm{Cl}$ molecule becomes attached first to the antibody through a specific component $\mathrm{Cl}_{\mathrm{q}}$ (Müller-Eberhard, 1968), fixation of which allows alterations to occur in the rest of the $\mathrm{Cl}$ molecule, with the formation of an esterase CIS which is capable of activating later components. The full classical sequence in relation to haemolysis is shown in figure 1 . Once activated, each component must become bound to its specific receptor, which may be either the previous component in the sequence or a nearby site on the red cell surface. The half-lives of these activated components can be very short indeed, of the order of milliseconds (Lachmann and Thompson, 1970). If the activated components fail to become bound they lose their binding sites and the capacity to activate later components in the sequence; they circulate in the plasma in their inactivated forms, which are of smaller molecular weight than their parent molecules, and are cleared from the circulation at varying rates.

At many of the steps within the complement sequence small molecular weight products are formed with specific physiological functions. Activation of $\mathrm{C} 3$ is by far the most important. Increased vascular permeability is caused by the formation of a low molecular weight peptide C3a (Ward, 1969), together with the generation of a chemotactic agent

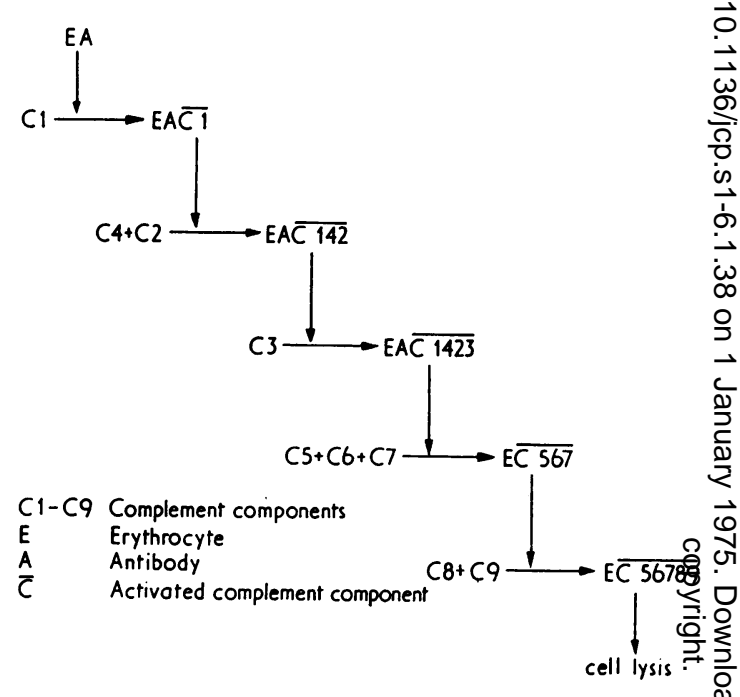

Fig 1 The classical complement pathway illustrated by erythrocyte lysis.

at the same step. It is now thought that the formation of these components and others of similar function is the most important role of the complement sequence, the formation of a lytic enzyme? being of less importance.

Such a potent enzyme system is under strict homeostatic control, and accidental triggering of $\delta$ the system, or the consequences of too great an activation, are controlled in one of two ways. First, $\frac{0}{2}$ the short half-lives of the activated components tend $>$ to limit the degree of activation. Secondly, there are inhibitors of the complement sequence within the circulation which act at three places within the ${ }^{\circ}$ sequence (fig 2). $\mathrm{Cl}$ inhibitor acts as a competitive $\tilde{O}$ inhibitor of C2 (Lepow, Ratnoff, and Levy, 1958), స్ల $\mathrm{C} 3$ inhibitor (KAF) inactivates $\mathrm{C} 3$ conversion to $\mathrm{C3c}$ and C3d (Tamura and Nelson, 1967), and C6 inhibitor prevents activation of $\mathrm{C} 7$ in a way which $\frac{\bar{D}}{\Phi}$ is not understood (Nelson and Biro, 1968). The full $\stackrel{?}{?}$ sequence of events, including the presence of 70 inhibitors and the phenomena induced by comple- $\bar{O}$ ment activation, is shown in figure 2 . 


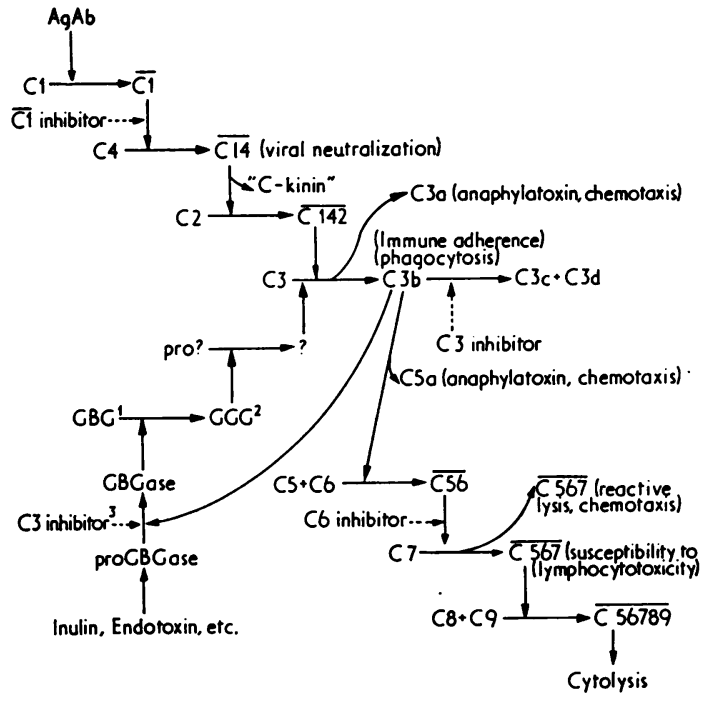

Fig 2 The classical pathway of complement activation from top left to bottom right, together with the alternative pathway linking in from the bottom left. It can be seen that conversion at $C 3$ and beyond can occur through the alternative pathway without much change in C4.

Complement activation has for many years been measured by the haemolytic complement-fixation test. The test is based on the haemolysis which occurs, in the presence of complement, of sheep red cells sensitized by specific antibodies raised in the rabbit. The amount of haemolysis is determined by the measurement of the haemoglobin released. Figure 3 shows the percentage lysis of sheep cells exposed to various concentrations of fresh guinea pig serum, ie, 'complement'. The dose response curve is clearly sigmoidal and reflects the complexity of the steps involved in the reaction. The slope is steepest around $50 \%$ lysis where small differences in haemolytic complement activity result in the greatest differences in the amount of lysis. For this reason $50 \%$ lysis (CH50) is taken as the endpoint in this test. The technique, however, requires well trained technical assistants, it is tedious and it requires carefully controlled conditions. Moreover, because of the complex nature of complement activation small differences in complement cannot be measured. Decreases in concentration of individual components of complement to $50 \%$ or less of the normal level may have little or no effect on this test. Reduction of haemolysis is usually associated with a drastic fall in the major component C3. Today the chief use of the $\mathrm{CH} 50$ in clinical medicine is to detect deficiency of a component of complement, a rare condition which results in the complete

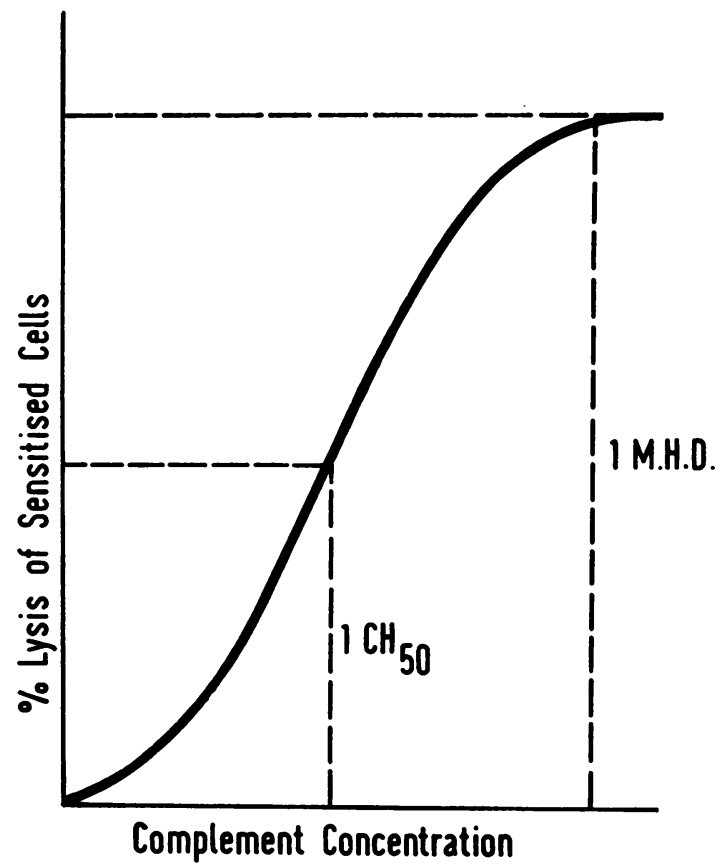

Fig 3 The relationship between percentage lysis of optimally sensitized sheep cells in one hour at $37^{\circ}$ and complement concentration. The greatest differences would occur at $50 \%$ lysis, ie, CH50.

absence of haemolytic activity. The complementfixation test has been superseded by immunochemical measurements of individual complement components, as antibodies have now been produced against most of the complement components. Antibodies against $\mathrm{C} 3$ and $\mathrm{C4}$, which account for $95 \%$ of total complement, are the most useful clinically, and are available at reasonable cost. Immunochemical estimation of the concentration of complement components may be carried out automatically by an automated immunoprecipitation system in which the light scattered at $357 \mathrm{~nm}$ is proportional to the amount of immune complex precipitated. This method is very precise, but the capital cost is high and only justified by a large work load. The two methods more satisfactory for smaller numbers are the Mancini technique of radial immunodiffusion (Mancini, Vaerman, Carbonara, and Heremans, 1964) and the Laurell 'rocket' electroimmunoassay (Laurell, 1966). Both these methods depend upon the migration of antigen into excess antibody dispersed uniformly within a gel matrix. Precipitation occurs at or near the equilibrium point as defined by Heidelberger and Kendall (1929). The distance of migration from the origin is proportional to the concentration of the 
antigen. Electroimmunoassay is to be preferred because of its greater accuracy, speed and sensitivity.

Measurements of complement components by these procedures cannot, however, distinguish between the amount of parent component and inactivated product, the levels of which are dependent on their relative rates of synthesis and catabolism. If these methods are used, only large changes in complement component are significant.

An increase in the serum concentration of a component of complement is generally not of major clinical importance. Most of the components behave as acute phase proteins and the levels, particularly of C3, may rise significantly in many inflammatory conditions, such as infections, rheumatoid arthritis, infarction, and after injury.

Low levels of complement components are much more important. These may result either from increased consumption (complement activation) or from decreased synthesis. Reduced C3 levels may be present in a wide variety of diseases such as acute glomerulonephritis and systemic lupus erythematosus, and transiently in acute viral hepatitis, serum sickness and some forms of drug reaction.

The level of C3 may be useful in diagnosis and in following the course of a disease. At the onset of acute glomerulonephritis the level of $\mathrm{C} 3$ is markedly reduced, but the level gradually returns to normal with recovery. Persistence of the low C3 levels in the plasma indicates that the disease is becoming chronic. The level of $\mathrm{C} 4$ may also be informative; in acute glomerulonephritis in particular, the $C 4$ is rapidly reduced to a very low level (Kohler and Bensel, 1969) but rapidly recovers and may be normal by the time the patient is admitted to hospital.

In systemic lupus erythematosus and other diseases in which soluble immune complexes have been demonstrated in the plasma, depression of the level of many complement components has been observed when the disease was severe, particularly when associated with renal involvement (Schur and Sandson, 1968); during the active phase $\mathrm{C1}_{\mathrm{q}}, \mathrm{C4}$, $\mathrm{C} 2$ and $\mathrm{C} 3$ have all been shown to be reduced (Hanauer and Christian, 1967). Serial observations have revealed depressed levels before the reappearance of clinical symptoms (Ruddy, Everson, Schur, and Austen, 1971). In active disease without renal involvement the level of $\mathrm{C4}$ may be reduced with normal levels of C3. Reduction of C4 generally precedes reduction of $\mathrm{C} 3, \mathrm{Cl}_{\mathrm{q}}$ and $\mathrm{CH}_{50}$ (Vaughan, Bayles, and Favour, 1951) and may take longer than these components to return to normal levels, once the attack subsides. In this disease the maintenance of normal complement levels is a desirable therapeutic goal.
In membranoproliferative or mesangio-capillary glomerulonephritis there is no evidence of antibody involvement. Some $50 \%$ of such patients exhibit low C3 levels, whilst the C4 level is invariably normal or raised (Gewurz, Pickering, Mergenhagen, $\stackrel{5}{\rightarrow}$ and Good, 1968a). The explanation of this will be? given later. The level of $\mathrm{C} 3$ may be extremely

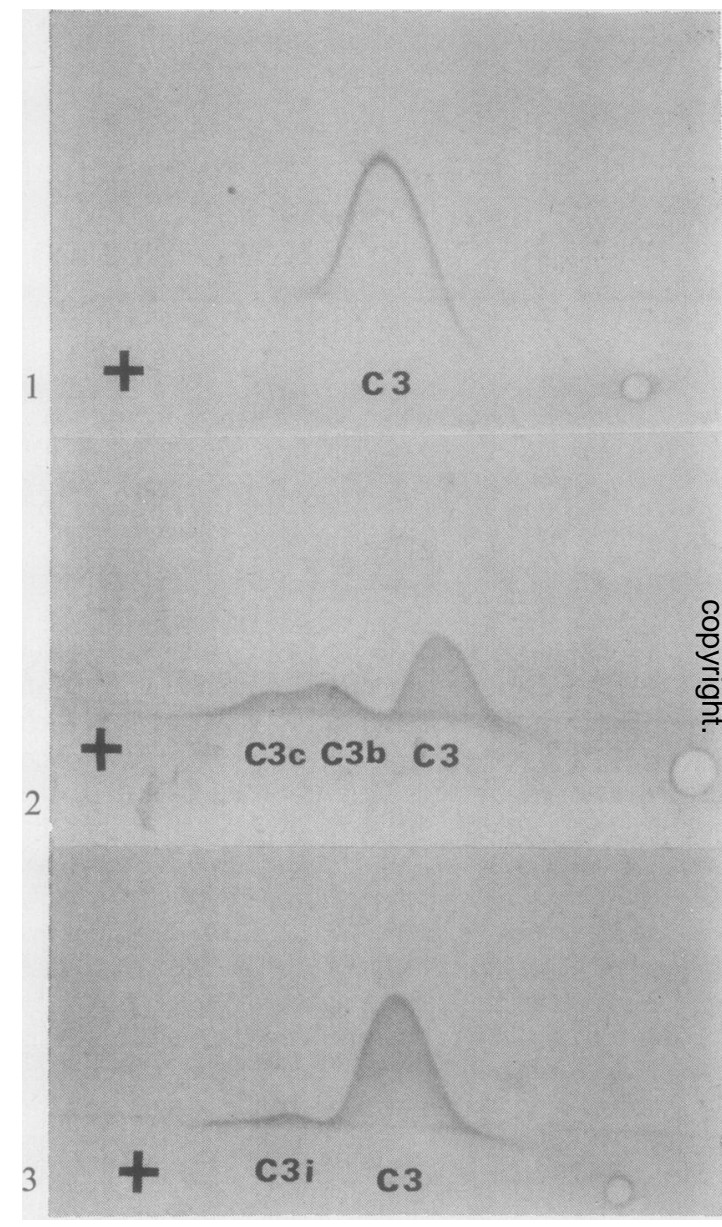

Fig 4 Three examples of two-dimensional immunoelectrophoresis of C3 in plasma. The plasma was applied in the well on the right and was first run towards the left and then at right angles into the agarose containing anti-C3. From above downwards are shown (1) normal plasma which has a single peak indicating that all the C3 is in its native form; (2) plasma from a patient with acute glomerulonephritis which shows a reduced $C 3$ level and the presence of two other peaks, $C 3 c$ and $C 3 b$, indicating that conversion of complement has taken place; (3) plasma from a patient with rheumatoid arthritis $C 3$ and small amounts of conversion products $(\mathrm{C} 3 i)$. 
variable and unrelated to disease activity (Cameron, Vick, Ogg, Seymour, Chantler, and Turner, 1973). Partial lipodystrophy can also be associated with a similar derangement of complement.

Both C3 and C4 may be low in severe haemolytic anaemia, septicaemia and some forms of liver disease (Grieco, Capra, and Paderon, 1971), particularly chronic active hepatitis and alcoholic cirrhosis. In the latter, decreased synthesis may be a factor.

Activation of complement results in the formation of inactive products of smaller molecular weight. These often have a different charge from their parent molecules and can be separated from them by electrophoresis. Immunoelectrophoresis (Graber, 1964) will show a line of identity between the parent molecule and the inactivated component. However, immunoelectrophoresis is not usually sufficiently sensitive to detect small levels of the inactive component. An allied technique described by Ressler (1960) and Laurell (1965) employing two-dimensionl immunoelectrophoresis increases the sensitivity. Samples separated by electrophoresis in agarose gel are electrophoresed at right angles to the previous separation into agarose gel containing specific antibody. Precipitation of specific antigen-antibody complexes occurs in the form of peaks. In fig 4 three such analyses are shown for C3. One shows the normal single peak of $\mathrm{C} 3$. Another, from a patient with acute glomerulonephritis, shows a reduced level of native $\mathrm{C} 3$ together with two smaller peaks, the inactivation products $\mathrm{C} 3 \mathrm{~b}$ and $\mathrm{C} 3 \mathrm{c}$. The third pattern, which is from a patient with rheumatoid arthritis, shows a normal level of $\mathrm{C} 3$ and only small amounts of $\mathrm{C} 3 \mathrm{~b}$ and $\mathrm{C} 3 \mathrm{c}$.

This technique is of particular importance in the investigation of many immune complex diseases such as acute glomerulonephritis, systemic lupus erythematosus, rheumatoid arthritis and ankylosing spondylitis. The presence of $\mathrm{C} 3$ activation or conversion is indicative of complement activation in vivo, and therefore provides non-specific evidence of disease activity, which is far superior to the ESR and other similar tests. Care, however, must be taken when interpreting results of such tests, since the method is extremely sensitive, and false positives may occur. All blood samples for such analyses should be taken into EDTA after discarding the first $2 \mathrm{ml}$ of blood drawn in which components from damaged tissue could have activated plasmin, which

PATIENT R.J.

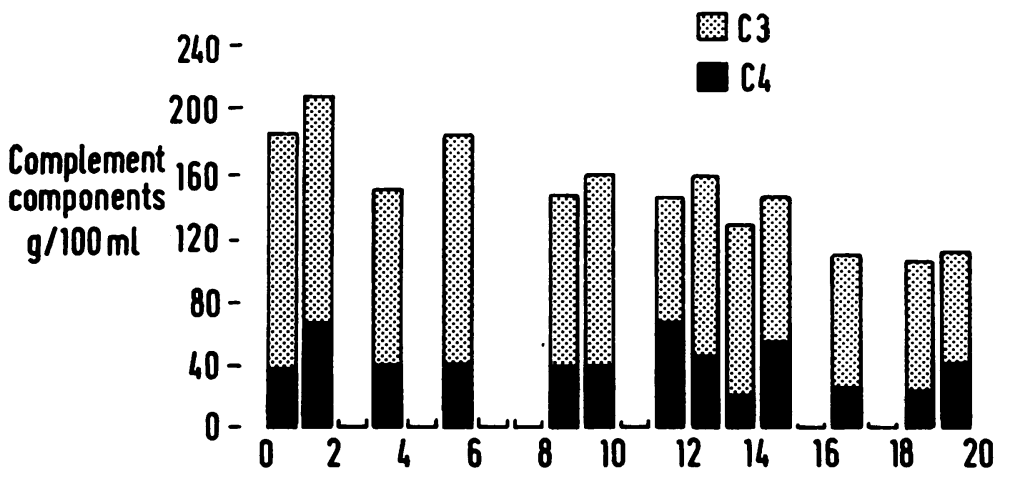

Fig 5 A patient with systemic lupus erythematosus in whom total levels of plasma C3 mask the degree of conversion which is actually occurring as shown by measurement of inactivation products.

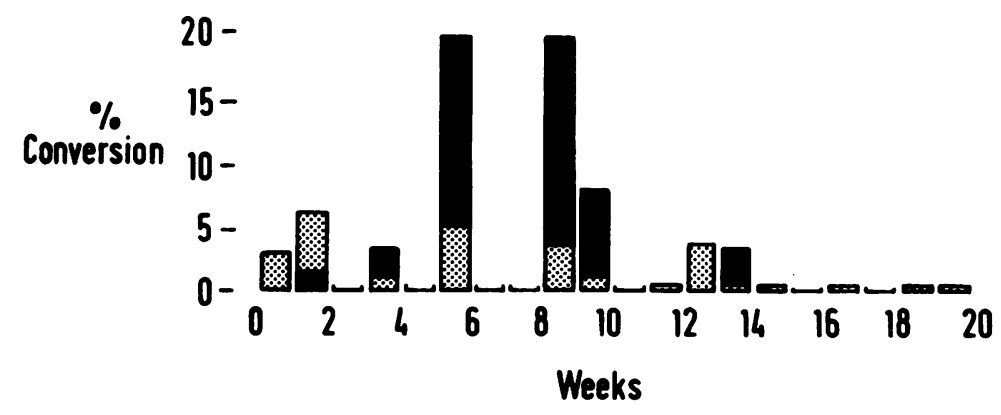


could then slowly convert complement after collection is completed.

Blood should be separated within two hours of collection and either electrophoresed immediately or stored at approximately $-70^{\circ}$. Electrophoresis should be performed in the presence of a buffer containing $1 \mathrm{mg} / \mathrm{ml}$ EDTA, since complement activation can be caused by the gel matrix itself.

Using this method it is possible to detect the presence of activation products of complement even when there is no fall in the level of the parent component. For example, fig 5 shows a patient with systemic lupus erythematosus without renal involvement; the $\mathrm{C} 3$ level gradually fell during the period of observation. During the course of treatment with prednisone, however, the patient relapsed, showing a peak of marked complement conversion. The dose of prednisone was increased, the level of conversion fell and the patient recovered. This illustrates one of the uses of complement conversion in the monitoring of treatment in immune complex diseases. In polymyalgia rheumatica, which responds quickly to prednisone treatment, normal levels of C3 may persist throughout the course of treatment, while the level of complement inactivation products falls rapidly after beginning treatment with prednisone.

Rheumatoid arthritis and ankylosing spondylitis, two other diseases in which immunological abnormalities have been described, may both exhibit complement conversion during periods of activity (Versey, Hobbs, and Holt, 1973). In fig 6 complement conversion is shown in such a patient with

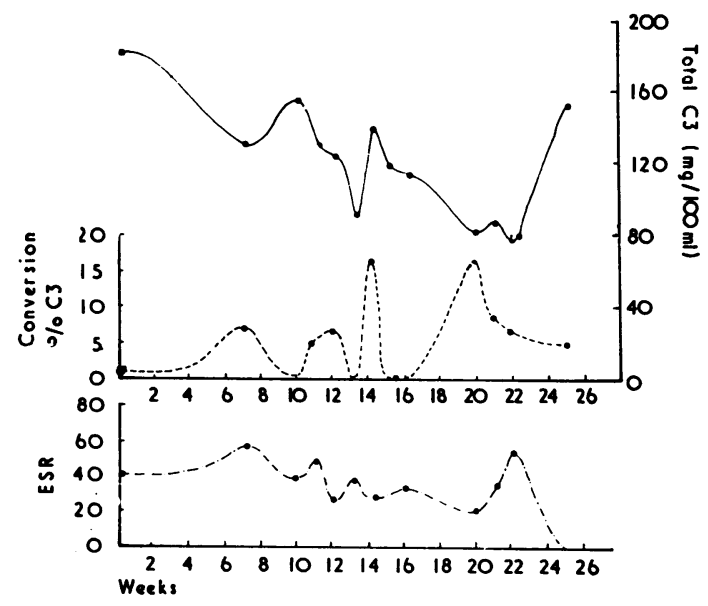

Fig 6 Serial levels of plasma $C 3$ and C3 conversion products and of ESR values in a patient with rheumatoid arthritis. Note intermittent peaks of conversion correlating with the ESR. active rheumatoid arthritis. The level of $\mathrm{C} 3$ is 0 extremely variable so that measurement of individual

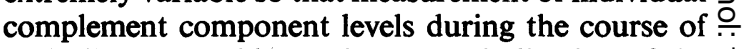
such disease would not give a true indication of the $\overrightarrow{\vec{D}}$ state of the patient.

In the preceding paragraphs, activation of the major component of complement, $\mathrm{C} 3$, has been $\frac{\bar{\sigma}}{\bar{c}}$. discussed. Many other components of complement, $\frac{\vec{\phi}}{\widehat{\phi}}$ when activated, also form products with electro- $\varrho$ phoretic mobilities which differ from that of the is parent molecule. $\mathrm{C} 4$ produces two major activation $\vec{\circ}$ products, one of slower and the other of faster mobility in agarose (Sjöholm and Laurell, 1973); $\vec{\omega}$ estimation of the faster products can prove useful in conjunction with $\mathrm{C} 3$ conversion. The level of $\mathrm{C} 4$ ? conversion produced in such a system is much lower is than that of $\mathrm{C} 3$ and may easily be missed. In diseases in which activation of complement involves $\vec{\omega}$ the full sequence, when for example, immune $\infty$ complexes are present, activation of $\mathrm{C} 4$ will parallel $\frac{\text { 의 }}{4}$ that of C3.

In addition to the classical sequence of complement activation, a new pathway of activity has recently been discovered. The early work of Pillemer and his coworkers (Pillemer, Blum, Lepow, Ross, $\vec{\varphi}$ Todd, and Wardlaw, 1954) established that a serum component, designated properdin, together wi certain cofactors, possessed the property of initiatins the complement sequence directly at the $\mathrm{C} 3$ leve when activated by a component of yeast cell walls, zymosan. This work was ignored for a decade, but observations have recently shown that a factor $\stackrel{\unrhd}{\varrho}$ isolated from cobra venom has similar properties $\overrightarrow{\vec{O}}$ (Müller-Eberhard and Fjellström, 1971). This $\frac{3}{3}$ component acts through a sequence of serumenzymes to form an enzyme capable of activating C3 directly, bypassing all earlier components (Nelson, 1966). Similar results have also been shown with many? other activators, including lipopolysaccarides or $\frac{5}{3}$ bacterial endotoxins, insulin and aggregated gamma globulin (Gewurz, Shin, and Mergenhagen, 1968b). This pathway of activity may be of more importance physiologically than the classical complement sequence. It has been suggested that the system may $\rightarrow$ be a major bacterial defence mechanism before antibodies are formed (Lachmann, Elias, and Moffet, $N$ 1972). Antibodies are not required for successful alternative pathway activity, which will result in $N$ some chemotactic and opsonin activity allowing a $\omega$ minor degree of phagocytosis of bacteria. For haemolysis, the alternative pathway is less efficient 6 than the classical pathway. Once antibodies are $\mathbb{\complement}$ formed the classical pathway will mask alternative $\stackrel{\mathcal{P}}{+}$ pathway activity. Estimation of the levels of indivi- $\frac{T}{0}$ dual components in the alternative pathway by $\frac{O}{\mathbb{D}}$ electroimmunoassay will give an indication of activity 
of the pathway. Antiserum to $\mathrm{C} 3$ proactivator or $\mathrm{GBG}^{1}$ (one of the components of the alternative pathway) is commercially available and can be used for its assay. Reduced levels give an indication of alternative pathway activity. Activation of GBG, like activation of $\mathrm{C} 3$, produces components of different electrophoretic mobility from the parent molecule (Boenisch and Alper, 1970). Demonstration of such activation by two-dimensional immunoelectrophoresis is a strong indication of alternative pathway activity, but is not easy because the two activation products may not separate sufficiently from the parent molecule for easy identification. In addition, since $\mathrm{C4}$ is not involved in the alternative pathway, the lack of $\mathrm{C} 4$ conversion in the presence of $\mathrm{C} 3$ conversion is a good indication of alternative pathway activity. Alternative pathway activity was illustrated by a patient who was bitten by a snake whose plasma revealed marked inactivation of C3 but not of C4 (Nicolson, Ashby, Johnson, Versey, and Slater, 1974). The scheme proposed for the alternative pathway by Lachmann is shown in figure 2.

Activation of the alternative pathway appears so far to be of limited clinical importance. Its significance has only been demonstrated in a few diseases. One notable example has been demonstrated in a patient described by Alper, Abramson, Johnston, Jandle, and Rosen, in 1970, who had suffered from severe recurrent infections since birth. Investigations revealed limited phagocytic activity which could be ascribed to a deficiency in complement activity. C3 levels were about one-third of normal. Two-dimensional immunoelectrophoresis revealed that three-quarters of this $\mathrm{C} 3$ was present in the inactivated form C3b. Furthermore no GBG could be detected. Recent work has shown that the primary deficiency is the lack of C3 inhibitor which normally prevents activation of GBG (Abramson, Alper, Lachmann, Rosen, and Jandl, 1971). This deficiency has been mimicked in vitro and its effects on the levels of complement components are very similar. Another family has recently been discovered at the Westminster Hospital. The twin patients both suffered from recurrent infections. Laboratory examination revealed that $\mathbf{T}$ and $\mathbf{B}$ cell lymphocyte functions were apparently normal. However, the patients appeared deficient in chemotactic factor like the patients described by Miller and Nilsson (1970). Examination of their humoral complement activity revealed that they had a normal complement level. Their $\mathrm{C1}_{\mathrm{q}}, \mathrm{C} 3, \mathrm{C4}$ and GBG levels were all normal. However, investigations showed that their ability to respond to bacterial lipopolysaccharides

1 Glycine-rich- $\beta$-glycoprotein was impaired, and that this block occurred somewhere in the alternative pathway sequence. If this is so, the importance of an intact alternative pathway system has been demonstrated. The patients' complement can be reconstitued both in vivo and in vitro by adding small amounts of fresh plasma. The patients have now had plasma therapy for six months. Their osteomyelitis and general health have much improved and there has been no sign of return of symptoms. In addition to these two known defects of the alternative pathway a third more common variant is known. In mesangiocapillary glomerulonephritis and partial lipodystrophy a factor can be isolated from the plasma of about $50 \%$ of patients which appears able to interact with C3 directly (Spitzer, Vallota, Forristal, Sudora, Stitzell, Davis, and West, 1970). The factor, NeF, is thought to be a separate activator of the alternative pathway, and may act as an antibody-like molecule to C3-inhibitor, thereby allowing alternative pathway activation. However, $\mathrm{NeF}$ has been isolated and characterized by Arroyave, Vallota, and Müller-Eberhard, 1974) and shown to be a protein but different from gamma globulin; it may have a counterpart in normal human serum.

Deficiencies of the complement system in man are very rare, and include reductions associated with impaired IgG synthesis (Kohler and MüllerEberhard, 1969), C1 ${ }_{\mathrm{r}}$ deficiency, C3 deficiency, partial C3 deficiency and C5 dysfunction, although the number of recorded cases is small. When the clinical features suggest a complement deficiency and a humoral defect can be demonstrated, the further investigation of such cases should be referred to a specialized laboratory.

So far no mention has been made of functional activity of the individual components. Methods of testing the latter have been described recently by Lachmann, Hobart, and Aston (1973); they cover all aspects of complement but are highly specialized and only available from a limited number of laboratories for individual patient studies on a research basis. When deficiency of complement is suspected analysis of total complement by $\mathrm{CH} 50$ tests will generally show whether any deficiency exists. When a deficiency has been proved, the examination should be completed in the more specialized laboratories.

\section{References}

Abramson, N., Alper, C. A., Lachmann, P. J., Rosen, F. S., and Jandl, J. H. (1971). Deficiency of C3 inactivator in man. J. Immunol., 107, 19.

Alper, C. A., Abramson, N., Johnston, R. B., Jr., Jandl, J. H., and Rosen, F. S. (1970). Increased susceptibility to infection associated with abnormalities of complement-mediated functions and of the third component of complement (C3). New Engl. J. Med., 282, 349. 
Arroyave, C. M., Vallota, E. H., and Müller-Eberhard, H. J. (1974). Lysis of human erythrocytes due to activation of the alternate complement pathway by nephritic factor (C3NeF). J. Immunol., $113,764$.

Boenisch, T., and Alper, C. A. (1970). Isolation and properties of a glycine-rich $\beta$-glycoprotein of human serum. Biochim. biophys. Acta (Amst.), 221, 529.

Cameron, J. S., Vick, R. M., Ogg, C. S., Seymour, W. M., Chantler, C., and Turner, D. R. (1973). Plasma C3 and C4 concentrations in management of glomerulonephritis. Brit. med. J., 3, 668.

Gewurz, H., Pickering, R. J., Mergenhagen, S. E., and Good, R. A. (1968a). The complement profile in acute glomerulonephritis systemic lupus erythematosus and hypocomplementaemic chronic glomerulonephritis: contrasts and experimental correlations. Int. Arch. Allergy, 34, 556.

Gewurz, H., Shin, H. S., and Mergenhagen, S. E. (1968). Interactions of the complement system with endotoxic lipopolysaccharide: consumption of each of the six terminal complement components. J. exp. Med., 128, 1049.

Graber, P. (1964). Immunoelectrophoretic analysis. In Immunological Methods edited, by J. F. Ackroyd, p. 79. Blackwell, Oxford.

Grieco, M. H., Capra, J. D., and Paderon, H. (1971). Reduced serum beta 1c/1a globulin levels in extrarenal disease. Amer. J. Med. 51,340 .

Hanauer, L. B., and Christian, C. L. (1967). Clinical studies of hemolytic complement and the $11 \mathrm{~S}$ component. Amer.J. Med. $42,882$.

Heidelberger, M., and Kendall, F. E. (1929). The quantitative study of the precipitin reaction between Type III pneumococcus polysaccharide and purified homologous antibody. J. exp. Med., 50, 1809.

Hobbs, J. R. (1971). Immunoglobulins in clinical chemistry. Advanc. clin. Chem., 14, 219.

Kohler, P. F., and Müller-Eberhard, H. J. (1969). Complementimmunoglobulin relation: deficiency of $\mathrm{C}^{\prime} 1 \mathrm{q}$ associated with impaired immunoglobulin G synthesis. Science, 163, 474.

Kohler, P. F., and ten Bensel (1969). Serial complement component alterations in acute glomerulonephritis and systemic lupus erythematosus. Clin. exp. Immunol., 4, 191.

Lachmann, P. J., Elias, D. E., and Moffet, A. (1972). In Biological Activities of Complement, edited by D. G. Ingram, 2nd ed., p. 202. Karger, Basle.

Lachmann, P. J., Hobart, M. J., and Aston, W. P. (1973). In Handbook of Experimental Immunology, edited by D. M. Weir, 2nd ed., ch. 5. Blackwell. Oxford.

Lachmann, P. J., and Thompson, R. A. (1970). Reactive lysis: the complement-mediated lysis of unsensitized cells. II. The characterization of activated reactor as C56 and the participation of C8 and C9. J. exp. Med., 131, 643-657.

Laurell, C. B. (1965). Antigen antibody crossed electrophoresis. Analyt. Biochem., 10, 358.

Laurell, C. B. (1966). Quantitative estimation of proteins by electrophoresis in agarose gel containing antibodies. Analyt. Biochem., $15,45$.

Lepow, I. H., Ratnoff, O. D., and Levy, L. R. (1958). Studies on the activation of a proesterase associated with partially purified first component of human complement. J. exp. Med., 107, 451 .
Macfarlane, R. G. (1964). An enzyme cascade in the blood clotting mechanism and its function as a biochemical amplifier: Nature (Lond.), 202, 498.

Mancini, G., Vaerman, J. P., Carbonara, A. O., and Heremans, J. F으 (1964). A single radial diffusion method for the immunologica quantitation of proteins. Protides, biol. Fluids, 11, 370.

Miller, M. E., and Nilsson, U. R. (1970). A familial deficiency of the phagocytosis enhancing activity of serum related to a dys-O function of the fifth component of complement. (C5). New Engl. J. Med., 282, 354-358.

Müller-Eberhard, H. J. (1968). Chemistry and reaction mechanisms of complement. Advanc. Immunol. 8, 1.

Müller-Eberhard, H. J., and Fjellström, K. E. (1971). Isolation of then anti-complementary protein from cobra-venom and its modes of action on C3. J. Immunol., 107, 1666.

Nelson, R. A., Jr. (1966). A new concept of immunosuppression in. hypersensitivity reactions and in transplantation immunity. Surv. Ophthalmol., 11, 498.

Nelson, R. A., Jr., and Biro, C. E. (1968). Complement-componentso of a haemolytically deficient strain of rabbits. Immunology $14,527$.

Nicolson, I. C., Ashby, P. A., Johnson, N. D., Versey, J. M. B., andids Slater, L. (1974). Boomslang bite with haemorrhage and activation of complement via the alternate pathway. Clin 0 exp. Immunol., 16, 295.

Pillemer, L., Blum, L., Lepow, I. H., Ross, O. A., Todd, E. W., and Wardlaw, A.C. (1954). The properdin system and immunity 1. Demonstration and isolation of a new serum protein, $\supset$ properdin and its role in immune phenomena. Science, 120, 279.

Ressler, N. (1960). Two-dimensional immunoelectrophoresis of protein antigens with an antibody containing buffer. Clin. chim. Acta, 5, 795 .

Ruddy, S., Everson, L. K., Schur, P. H., and Austen, K. F. (1971) Hemolytic assay of the ninth complement component : elevation and depletion in rheumatic diseases. J. exp. Med., 134, Suppl., $\vec{\theta}$ 259.

Schur, P. H., and Sandson, J. (1968). Immunologic factors clinical activity in systemic lupus erythematosus. New Eng J. Med., 278, 533.

Sjöholm, A. G., and Laurell, C-B. (1973). Conversion of the fouth complement component studied by crossed immunoelectro $-\bar{O}$ phoresis. Clin. exp. Immunol., 14, 515.

Spitzer, R. E., Vallota, E. H., Forristal, J., Sudora, E., Stitzel, A.@̊ Davis, N. C., and West, C. D. (1969). Serum C3 lytic system in patients with glomerylonephritis. Science, 164, 436.

Tamura, N., and Nelson, R. A., Jr. (1967). Three naturally occurringō inhibitors of components of complement in guinea pig andB rabbit serum. J. Immunol., 99, 582.

Vaughan, J. H., Bayles, T. B., and Favour, C. B. (1951). Serum complement in rheumatoid arthritis. Amer. J. med. Sci., 222 . 186.

Versey, J. M. B., Hobbs, J. R., and Holt, P. J. L. (1973). Complement? metabolism in rheumatoid arthritis. I. Longitudinal studies. Ann. rheum. Dis., 32, 557.

Ward, P. A. (1969). The heterogeneity of chemotactic factors for neutrophils generated from the complement system. In Cellular and Humoral Mechanisms in Anaphylaxis and Allergy, edited by H. Z. Morat, p. 279, Karger, Basle. 